WG5 / CLEO $/$ Pacific Rim '99/ 183

\title{
Monolithic PPLN-based Optical Parametric Oscillators Using Grating Structures for Phase Compensation in Achieving Efficient Round-trip Wavelength Conversion
}

\author{
Chih-Wei Hsu, Cheng-Yen Chen and C. C. Yang \\ Institute of Electro-Optical Engineering, National Taiwan University, \\ 1, Roosevelt Road, Sec. 4, Taipei, Taiwan, R.O.C. \\ (phone) 886-2-23657624 \\ (fax) 886-2-23652637 \\ (E-mail) ccy@cc.ee.ntuedu.tw
}

With the development of domain inversion techniques for quasi-phase matched $\mathrm{LiNbO}_{3}$ and other nonlinear optical materials, wavelength conversion efficiencies for frequency doubling and optical parametric processes have been tremendously increased [1]. However, so far all oscillators based on quasi-phase-matching (QPM) materials for wavelength conversion were made with the configuration of external cavity. It is a natural trend to develop compact monolithic wavelength converter. Such a device can be implemented by preparing high-reflection coating on the end-facets of a QPM material to reduce the oscillation threshold. However, such an arrangement faces a basic problem, i.e., the length of the last QPM segment at the material end facet is uncontrollable because the period of QPM ranges from a few $\mu \mathrm{m}$ to a few tens $\mu \mathrm{m}$. Unless the length of the last QPM period is exactly equal to one-quarter of the QPM period (the optimum situation), the reflected signal from the end-facet will experience broken QPM periodicity and suffer from reduced wavelength conversion efficiency or even reverse energy flow of wavelength conversion [2]. This problem can possibly be solved by preparing anti-reflection coating for one of the pump, signal and idler waves. However, since high-reflection coating needs to be prepared for the other one or two waves at the same time to reduce the oscillation threshold, the coating process becomes quite difficult.

In this paper, we propose the idea of using a grating structure for solving the problem of the incomplete QPM period and demonstrate the simulation results of the phase compensation in achieving efficient round-trip optical parametric oscillation in PPLN. Figure 1 shows the layout of the a monolithic optical parametric oscillator (OPO) with two surface gratings at both ends. The two gratings are chirped to guarantee that their phase variations over the Bragg reflection windows are larger than $2 \pi$. One of them has a length of $1 \mathrm{~mm}$ and the other has a length of $0.8 \mathrm{~mm}$. The pump input is applied from the long grating side and the signal output is obtained from the short grating side. The whole PPLN length is $5 \mathrm{~mm}$. For our numerical study, we consider the pump wavelength at $1064 \mathrm{~nm}$ and signal wavelength at $1550 \mathrm{~nm}$. The idler wavelength is hence at $3.393 \mu \mathrm{m}$. The corresponding QPM period of PPLN is $29.6 \mu \mathrm{m}$. The central grating period is $361.83 \mathrm{~nm}$, which corresponds to the Bragg wavelength $1550 \mathrm{~nm}$. To obtain numerical results, we solved the coupled equations for the forward and backward pump complex amplitudes, the forward and backward signal complex amplitudes, and the forward and backward idler complex amplitudes.

The symbolic data points in Fig. 2 (3) show the output signal intensity as a function of the signal wavelength within the Bragg reflection window for various $L_{i}$ values of the grating of $1(0.8) \mathrm{mm}$. Here, $L_{i}$ is the length of the last QPM segment in terms of the QPM period $\Gamma$. Before the grating, there is a plain PPLN of 4 (4.2) $\mathrm{mm}$ in length. At the crystal end, the signal experiences uncoated end-facet reflection besides the Bragg reflection from the grating. Meanwhile, the idler and the residual pump are reflected from the uncoated and high-reflection (100\%) end-facets, respectively. For comparison, the data curves without symbols in Figs. 2 and 3 show the similar cases without the grating sections. In these cases, the end-facet is high-reflection coated for the signal. The pump intensity is fixed at $500 \mathrm{MW} / \mathrm{cm}^{2}$. The initial signal and idler intensities at the left end are set at 0 and $1.25 \mathrm{~kW} / \mathrm{cm}^{2}$, respectively. We can see that with the grating the output signal intensity oscillates with wavelength. The maximum of the oscillation reaches the level of the optimum situation of the coating device and the minimum has a level about the same as the worst situation of the coating device. The oscillating output signal intensity is attributed to the signal phase variation with wavelength upon reflecting from the grating. At the peaks, the phase of the reflected signal has a value such that the phase-matching condition among the reflected pump, signal and idler is reached for the optimum nonlinear conversion. The results in Fig. 2 imply that with the grating structure, the system will provide the output with the optimum nonlinear conversion by slightly shifting the signal wavelength within the Bragg reflection window of the grating. The wavelength shift is quite small, as small as a few tenths of nm. Such a shift is always within the QPM window 
for efficient nonlinear conversion. Because of the difference in grating length between Figs. 2 and 3 , the wavelengths for optimum wavelength conversion are different.

Then, we conducted numerical simulations on the whole PPLN with the two gratings. It was observed that the wavelength for optimum OPO signal output resulted from the compromise between the optimum conditions of the two gratings. It also depended on various conditions, such as the input pump power and the length of the central portion of the PPLN resonator. The details of the compromise for the optimum condition and the dependencies on various conditions will be discussed in this paper. Regarding the technique of forming the surface gratings, although chemical wet etching is not a good technique for fabricating corrugation gratings on periodically poled nonlinear crystals because the etching rates of the opposite domains are different, surface gratings on $\mathrm{LiNbO}_{3}$ have been successfully fabricated with laser ablation. Figure 4 shows an atomic force microscopy picture of a surface grating on $\mathrm{LiNbO}_{3}$. The details of this technique will also be discussed.

\section{References:}

1. G. D. Miller, R. G. Batchko, W. M. Tulloch, D. R. Weise, M. M. Fejer and R. L. Byer, Opt. Lett. 22, 1834 (1997).

2. G. Imeshev, M. Proctor and M. M. Fejer, Opt. Lett. 23, 165 (1998).

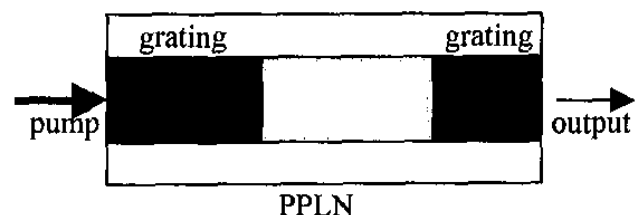

Fig. 1 Layout of the PPLN OPO with surface gratings.

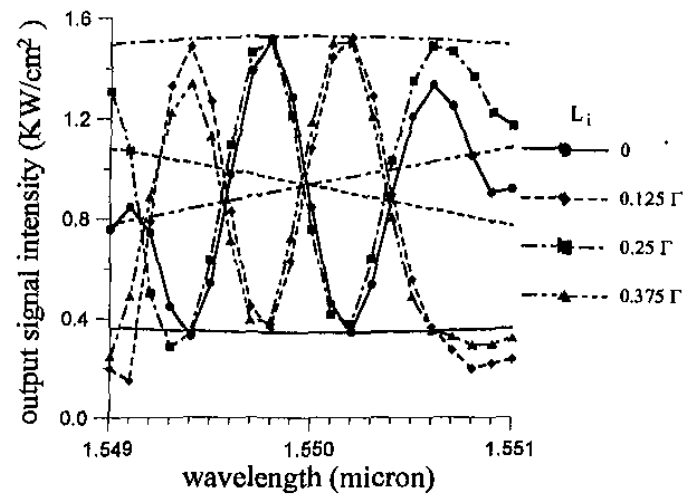

Fig. 3 Output signal intensity versus $L_{I}$ of the short grating.

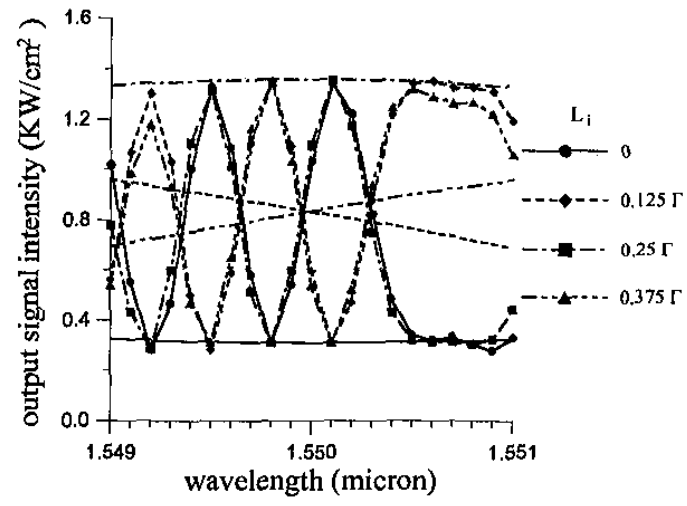

Fig. 2 Output signal intensity versus $\mathrm{L}_{\mathrm{I}}$ of the long grating.

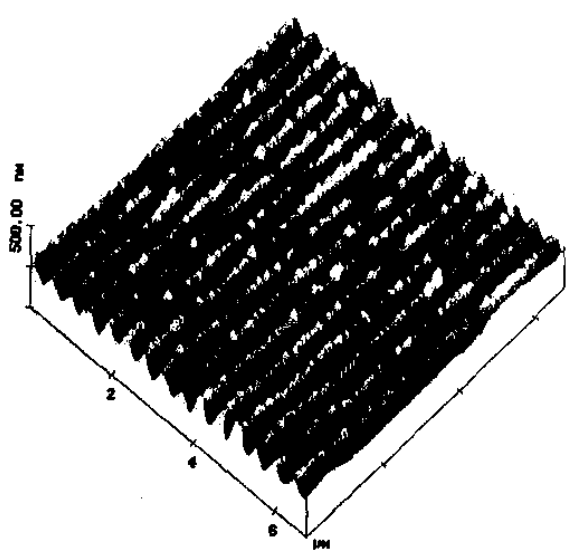

Fig. $4 \mathrm{AFM}$ picture of a $\mathrm{LiNbO}_{3}$ surface grating. 